\title{
6 Morphological Mutations
}

Alterations in the morphological pattern of any type, through mutagens, are regarded as morphological mutations. Morphological mutations affecting important plant attributes in many cases prove to be promising from breeder's point of view. Morphological mutants play a vital role in modifying the characteristics of cultivars for the construction of ideotype, and ultimately, lead to development of new variety of crops. For the development of improved varieties such mutants were found to be more productive, when used in cross breeding (Pawar et al., 2000). Several morphological mutants based on different plant forms, leaf, maturity, pod and seed characters were reported with the application of single and combination treatments of physical and chemical mutagens in pulses (Blixt, 1972; Appa Rao and Reddy, 1975; Filippetti and De Pace, 1986; Jha, 1988; Vanniarajan et al., 1993; Venkatachalam and Jayabalan, 1995; Ramesh and Dhananjay, 1996; Ravikesavan et al., 2001; Henry, 2002; Khan et al., 2005b; Solanki, 2005; Singh, 2007b; Selvam et al., 2010, Khan and Tyagi, 2010a; Wani et al., 2011a), cereals (Okuno and Kawai, 1978; Reddy and Gupta, 1988; Reddy, 1992; Singh et al., 1998; Viswanatahan and Reddy, 1998; Kumar and Mani, 1977; Ali and Siddiq, 1999, Singh, 2006) and other plants of economic importance (Chaghtai and Hasan, 1980; Zeerak, 1990; Datta and Laxmi, 1992; Marry and Jayabalan, 1995; Kumar et al., 1996; Amarnath and Prasad, 2000; Datta and Sengupta, 2002; Cagirgan, 2006). In chickpea, like other pulse crops, morphological mutations have been isolated for flower colour and structure, growth habit, size and colour of the seeds (Khosh-Khui and Niknejad, 1971; Muehlbauer and Singh, 1987; Davis et al., 1990; Davis, 1991; Ahmed and Godward, 1993; Ghate, 1993; Knight, 1993; Pundir and Reddy, 1998; Gaur and Gour, 2001; Toker and Cagirgan, 2004b; Toker, 2009; Khan et. al, 2004 \& 2011). The presence of more than one mutation in a single plant was termed as „multiple mutation” by Sharma (1969). According to him, agents with higher mutagenic efficiency induce more multiple mutations, and such mutations may accumulate several desirable mutations within one plant. Multiple mutations have been reported earlier by Kharkwal (1999) in Cicer arietinum, Appa Rao et al. (1975) in Vigna mungo, Odeigah et al. (1998) in Vigna unguiculata, Auti and Apparoa (2009) in Vigna radiata.

Tyagi and Gupta (1991) reported that each gene, which is of agronomic interest can mutate and, hence, a wide spectrum of viable mutants, morphological in nature, can be expected in mutation experiments. Based on segregation pattern of morphological mutants, Reddy and Gupta (1988) and also Thakur and Sethi (1993) observed that most of the true breeding mutants were conditioned by single recessive genes, however, Konzak et al. (1969) argued that the different morphological mutants which bred true in future generations like tall, dwarf, semidwarf, bushy, prostate and bold seeded mutant types were found to be under the influence of polygenes. 
Frequency of morphological mutations has been found to increase with increases in the dose of mutagen (Thakur and Sethi, 1995). Datta and Sengupta (2002) reported that spectrum of viable mutations was wider at lower doses of mutagens. Vaniranjan et al. (1993) observed the higher frequency of viable mutations at medium doses of gamma rays and EMS treatments. Thus, the spectrum and frequency of morphological mutations vary with mutagen and duration of treatment (Kumar and Mani, 1997) and the genetic differences of the experimental organism, which have a key role in the recoverable frequency and spectrum of morphological mutations (Kharkwal, 1999; Sharma, 2001; Khan et al., 2004b).

\subsection{Case Study 4}

In any mutation breeding programme, isolating and characterizing the morphological mutants in $\mathrm{M}_{2}$ generation progenies plays an important role. The morphological mutants play an important role in selection of desired characteristics in any hybridization programmes. In the present study as revealed in past chapters in Case Studies 1-3 some of the morphological mutants with their characteristics features and some physiological studies associated are discussed.

\subsubsection{Results}

A wide range of morphological mutants was isolated in the population of two varieties of chickpea in $M_{2}$ generation (Figs. 6.1-6.5). These mutants involved traits affecting plant height, growth habit, leaf, flower and pod. Mutation frequency was calculated on $\mathrm{M}_{2}$ plant basis. The highest frequency was noted in EMS treated population and the lowest with gamma rays treatments, while combination treatments of gamma rays + EMS were intermediate (Fig. 6.1). The frequency of morphological mutations was higher in the variety Pusa-256 than the variety BG-1053. The spectrum of mutations induced by EMS was comparatively wider than that of gamma rays and the combined gamma rays + EMS treatments in both varieties. Mutations affecting growth habit and leaf appeared more frequently followed by flower, plant height and pod in both the varieties (Fig. 6.1). In general, tall, broad/giant leaves, elongated rachis, double flower, open flower and bold pod mutants were predominant at the lower doses of gamma rays and EMS employed alone or in combination. In the two varieties of chickpea, the combined treatments of gamma rays and EMS did not produce additive effects on morphological mutations. A brief description of the morphological mutants identified is given below: 

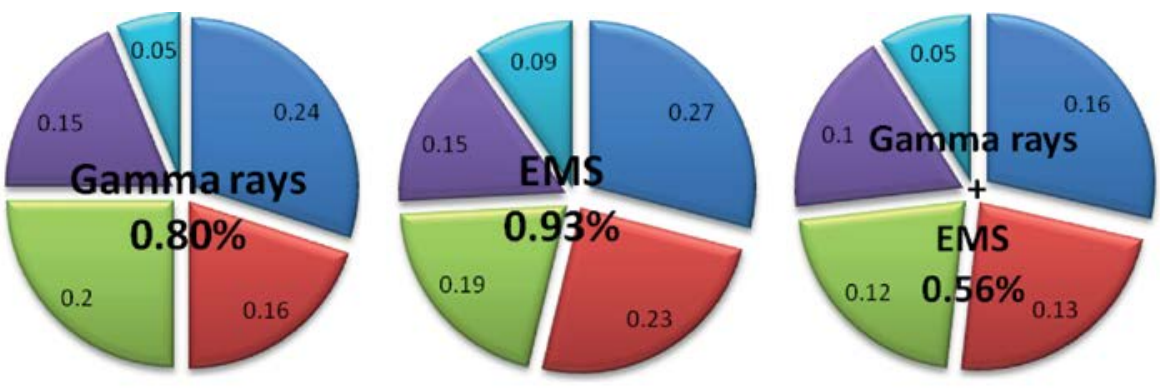

(Mutagen pooled over varieties)
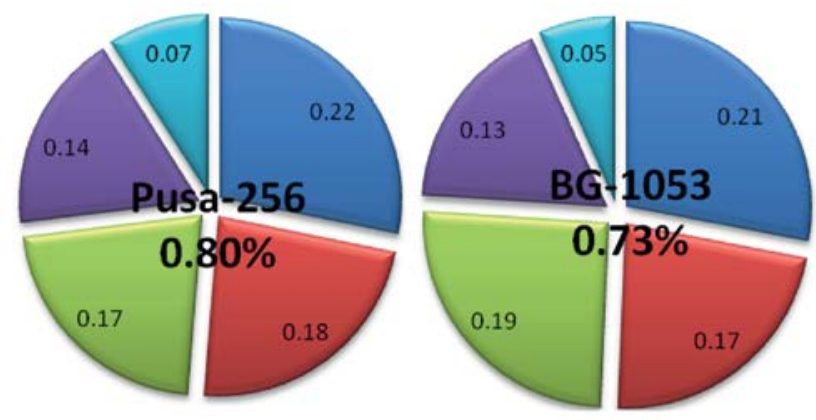

(Varieties pooled over mutagens)

a plant height

Growth habit

Leaf

Flower

a Pod

Fig. 6.1: Total (pooled) frequency and spectrum of morphological mutants induced in $M_{2}$ generation of chickpea.

\section{Mutants with altered plant height}

(i) Tall mutants: These mutants were observed at lower doses of single and combination treatments of gamma rays and EMS in both varieties. The mutants were considerably taller than the control plants, their height being $73-78 \mathrm{~cm}$ whereas it was 54-57 cm for the control plants. The pods and seed setting were low (Fig. 6.2-1).

(ii) Dwarf mutants: These mutants were isolated from the higher doses of the mutagens in both the varieties except in combination treatments of gamma rays and EMS in the Pusa-256. These mutants had short internodes and almost all yield components were reduced. Seeds were smaller in size. The plant height ranged from 20 to $25 \mathrm{~cm}$ (Fig. 6.2-2). 

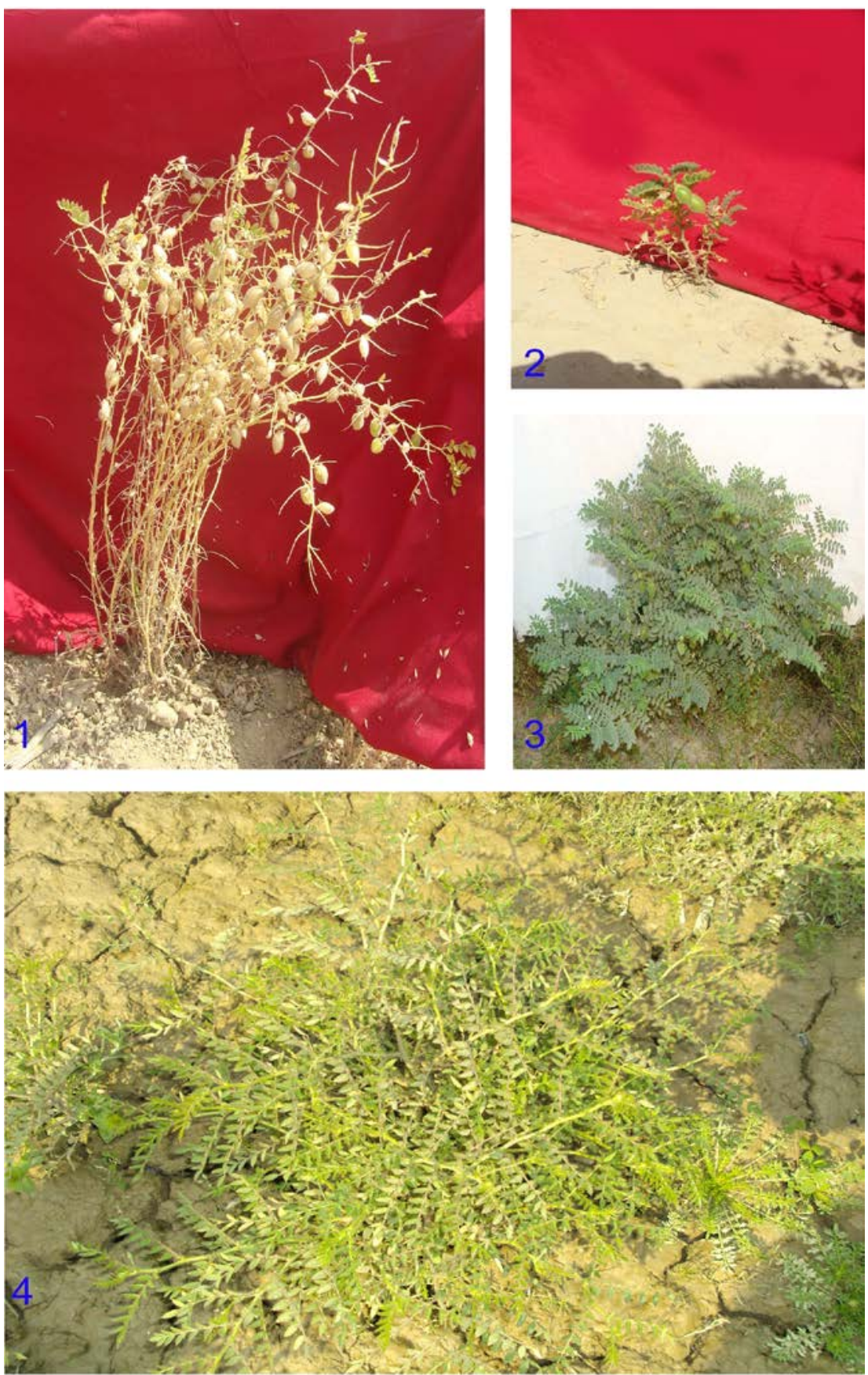

Fig. 6.2: Morphological mutants (mutants with altered plant height and growth habit): 1 . Tall mutant; 2. Dwarf mutant; 3. Bushy mutant; 4. Prostrate mutant (Photographs by M I Kozgar) 


\section{Mutants with altered growth habit}

(i) Compact/Bushy mutants: These mutants exhibited vigorous growth. Branches were short and the plant height was reduced giving it a bush like appearance. Yield per plant, more or less, was equal to the control plants. The mean height of the mutant was $35.40 \mathrm{~cm}$. These mutants were isolated at higher doses of mutagens in both varieties (Fig. 6.2-3).

(ii) Prostrate mutants: These mutants had long internodes and failed to grow erect with trailing branches at the ground level. The mutants possessed small pods containing 1-2 shrivelled seeds with hard and rough seed coat. They were isolated from the combined gamma rays + EMS treatments in both varieties (Fig. 6.2-4).

(iii) Spreading mutants: These mutants were isolated at $300 \mathrm{~Gy}$ gamma rays in the variety Pusa-256 and 0.4\% EMS in the variety BG-1053. These had long internodes and large canopy. The branches appeared to emerge erect separately at the ground. The leaves were mostly of brad type and the yield was reduced (Fig. 6.3-4).

(iv) One sided branching mutants: All branches were produced on one side of the stem. Plants were late in flowering, partially sterile with few pods only, seeds extremely shriveled, dark and non-viable. These mutants were isolated in the treatments with gamma rays in both the varieties and in combination treatments of $100 \mathrm{~Gy}$ gamma rays $+0.2 \%$ EMS in the variety BG-1053 (Fig. 6.3-3).

(v) Axillary branched mutants: Mutant plants were characterized by profuse axillary branches, short internodes, small pods, low seed setting and seeds were smaller in size. These mutants were noticed at higher doses of mutagens used in both varieties (Fig. 6.3-2).

\section{Mutants with altered leaf morphology}

(i) Broad/Giant leaves mutants: These mutants were tall and vigorous with large, thick leaflets. The size of stipules was larger than the normal stipules in control plants. Number of pods increased and they had bold seeds. These mutants were isolated at the lower and moderate doses of gamma rays + EMS employed alone or in combination in both varieties (Fig. 6.4-3).

(ii) Narrow leaves mutants: These mutants had small, narrow leaves with pointed tips. Pods were smaller in size, with a reduced number of seeds. Seed size was smaller and they were dark in colour. They appeared at higher doses of the mutagens in both varieties (Fig. 6.4-2).

(iii) Altered leaf architecture mutants: Mutant plants were characterized by vast variation in the leaf margins and apices. Variations in the plant heights were also noticed and such mutants were $5-7 \mathrm{~cm}$ shorter than the control plants. These mutants were isolated in almost all the mutagenic treatments (Figs. 6.4-4 and 6.4-5).

(iv) Elongated rachis: The mutants were characterized by elongated rachis with reduced number of leaves. These mutants were isolated at lower doses of mutagens in both varieties (Fig. 6.4-7). 

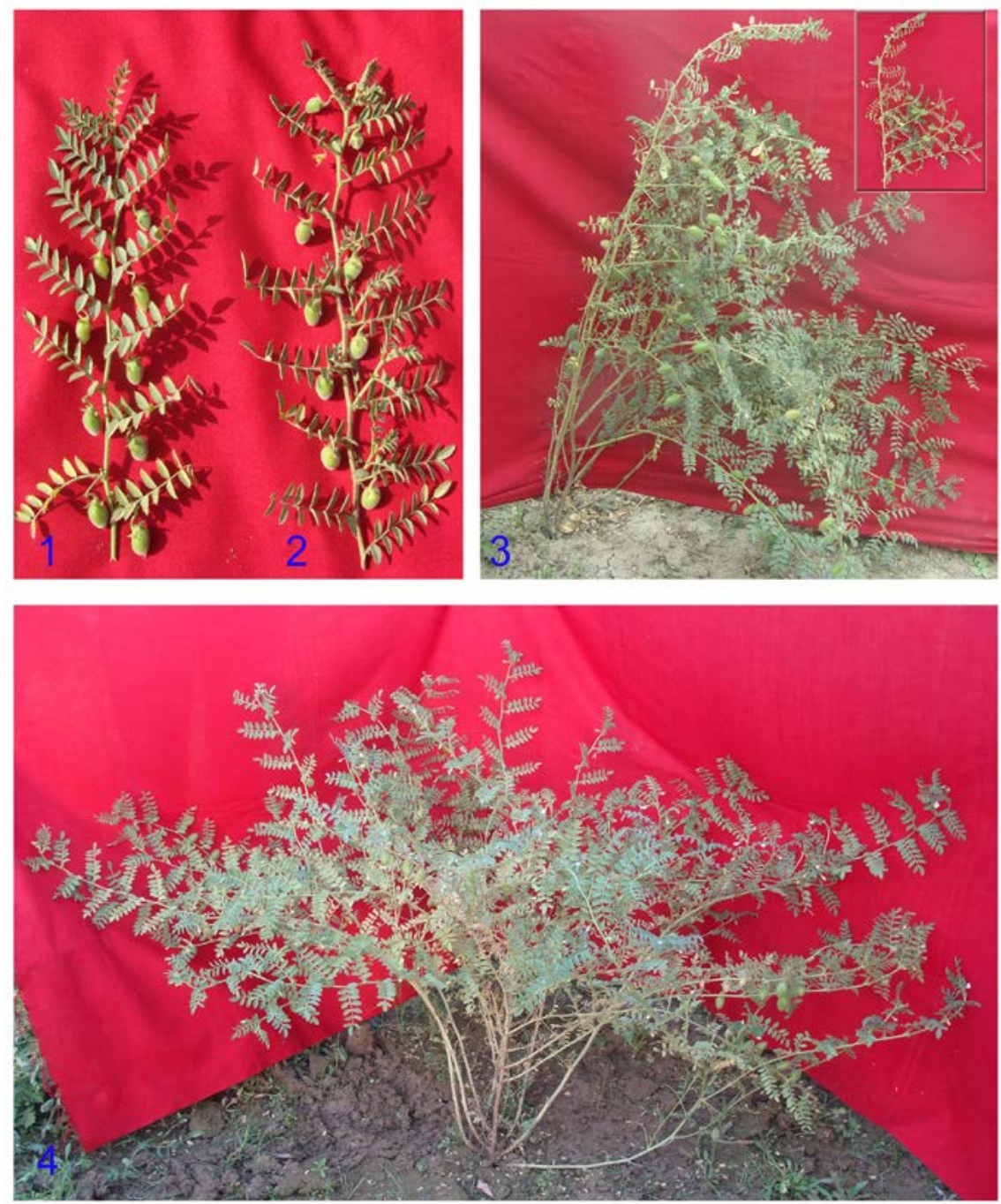

Fig. 6.3: Morphological mutants (mutants with altered growth habit): 1. Control; 2. Axillary branched mutant; 3. One sided branching mutant; 4. Spreading mutant (Photographs by M I Kozgar)

\section{Mutants with altered flower characters}

(i) Double flower mutants: These mutants were characterized by the presence of two flowers borne on the same stalk (peduncle) in contrast to one in control. The leaflets, pods and seeds were smaller in size (Fig. 6.5-3). The plants were normal in growth but late in flowering. These mutants appeared at $0.1 \%$ of EMS treatments in the variety Pusa-256.

(ii) Flower colour mutants: In the variety Pusa-256 white colour flower mutants were noticed (Fig. 6.5-2), in comparison to pink colour flower in the control, in $400 \mathrm{~Gy}$ gamma rays treatments. As compared to white colour flower in control, light blue 

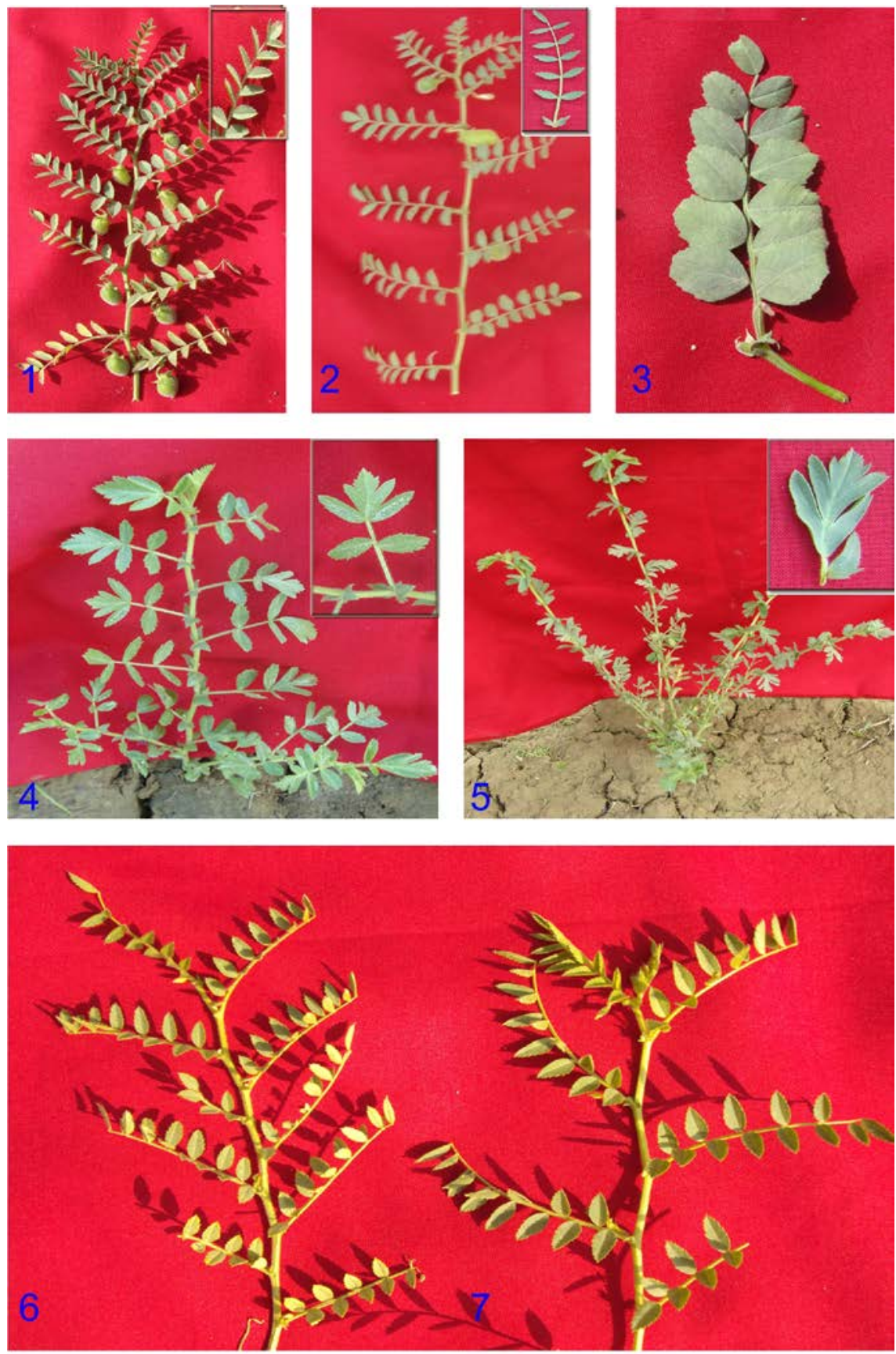

Fig. 6.4: Morphological mutants (mutants with altered leaf morphology): 1. Leaf of control plant; 2. Narrow leaf (small narrow leaflets with pointed tips); 3. Giant leaf mutant (large and thick leaflets, larger size stipules); 4. \& 5. Altered leaf architecture mutants; 6. Rachis (Control); 7. Elongated rachis mutant (Photographs by $M I$ Kozgar) 

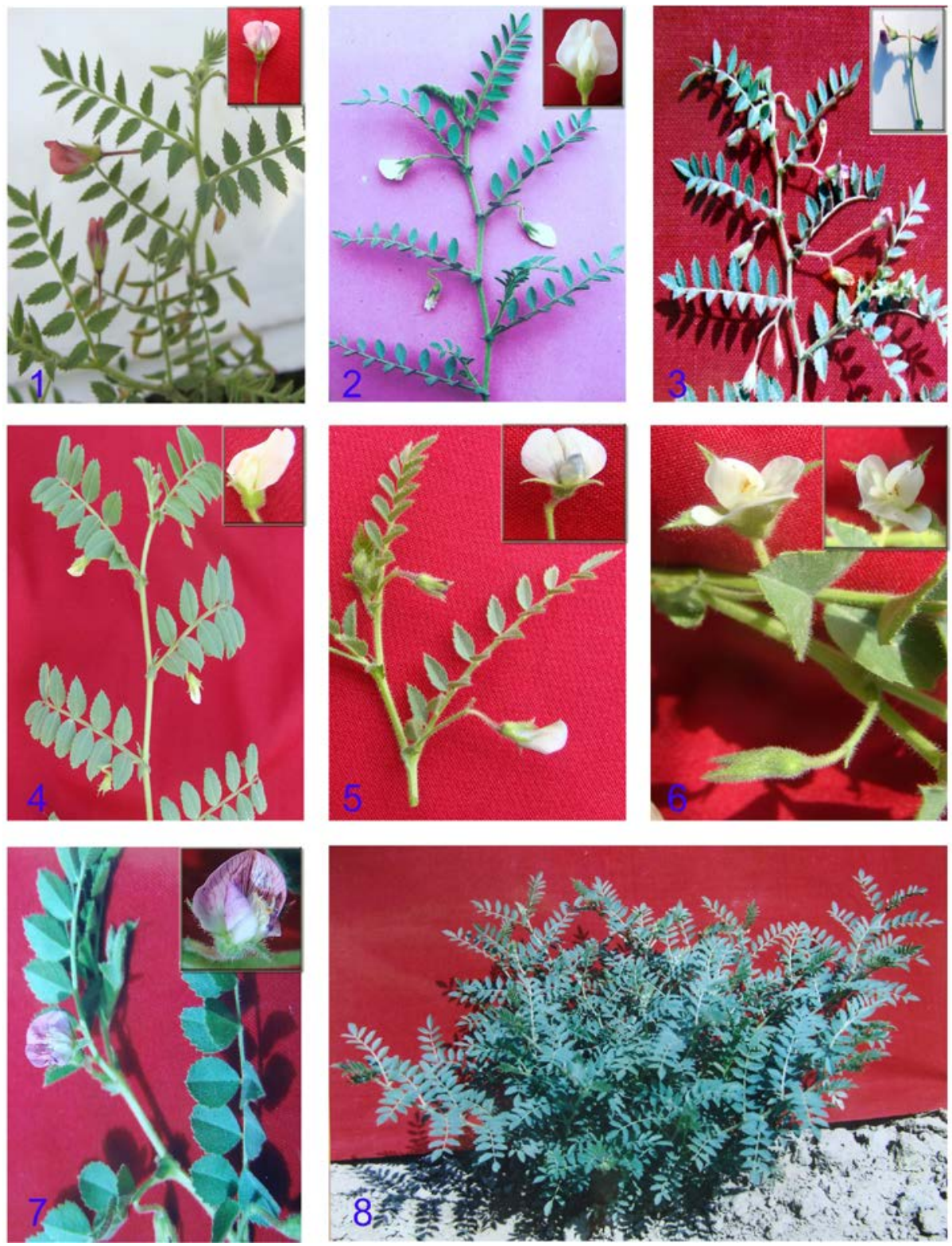

Fig. 6.5: Morphological mutants (mutants with altered flower characters): 1. Pink colour flower (Pusa256-Control); 2. White flower mutant of Pusa-256; 3. A branch showing double flowers (Pusa-256); 4. White colour flower (BG-1053-Control); 5. Light blue flower mutant of BG-1053; 6. Open flower mutant of BG-1053 (Androecium open); 7. Open flower mutant of Pusa-256 (Androecium and Gynoecium open); 8. Non-flowering/Vegetative mutant (Photographs by M I Kozgar) 
colour flower mutants were found in combination treatments of $200 \mathrm{~Gy}$ gamma rays + $0.2 \%$ EMS in the variety BG-1053 (Fig. 6.5-5).

(iii) Open flower mutants: These mutants possessed bell-shaped flowers with open keel and wings. The androecium and gynoecium were exposed (Fig. 6.5-6 and 7). Flowers withered without fruiting. They appeared at the lower concentrations of EMS in both varieties.

(iv) Non-flowering/Vegetative mutants: These mutants did not bear flowers and continued to grow vegetatively by the time other plants were setting seeds. They had profuse branching, dark green foliage, short internodes and large leaves (Fig. 6.5-8). These mutants were noticed in lower doses EMS and combination treatments.

(v) Late flowering mutants: In these plants, the flowering started 8-10 days later and growth period was extended by a similar number of days from the expected normal 95 days. These mutants were isolated at the higher doses in both single and combination treatments.

(vi) Early maturing mutants: These mutants matured 10-15 days ahead of their respective controls, of around 145 days. Early maturing mutants were recorded at the lower doses of the mutagens in both varieties.

\section{Mutants with altered pod characters}

(i) Small/Narrow pods: These mutants were mostly found at the higher doses of mutagenic treatments, except gamma rays and combination treatments in the variety BG-1053. These were characterized by presence of narrow leaves. In these mutants yield per plant was lower than in the control.

(ii) Bold pod mutants: The mutant plants exhibited vigorous growth and pods were larger containing 2-3 normal seeds. As a result of bold pods, a significant increase in yield was recorded. These mutants were also evaluated up to $M_{3}$ generation. The mutants were observed at the lower doses of the single and combination treatments of gamma rays and EMS.

\subsubsection{NRA, Chlorophyll and Carotenoid Contents of Morphological Mutants}

The alterations in the NRA assay, chlorophyll and carotenoid contents due to the mutagenic treatments of gamma rays, EMS and their combinations in the leaves of morphological mutants indicate that the activity of nitrate redutase (NR) and the contents of the chlorophyll and carotenoid vary differently with the mutant type (unpublished data). Among the mutants the highest activity of NR and high carotenoid contents have been found in bold seed pod mutants in both varieties of chickpea. The high chlorophyll content was also detected in the bold pod mutants in the variety Pusa-256, whereas in the variety BG-1053 the high chlorophyll content was detected in early flowering mutants. The values of all the three parameters (NRA, chlorophyll and carotenoid contents) mostly differed significantly between the mutant types and also from the control values either in positive or negative directions. 


\subsubsection{Discussion}

Enhancement of frequency and spectrum of mutations in a predictable manner for achieving desired plant characteristics is an important goal of induced mutagenesis programmes for the improvement of crop productivity. In the present study, the morphological mutations affecting different plant characteristics were isolated basing on the screening of $\mathrm{M}_{2}$ population. These mutants differed from control in plant height, growth habit, leaf, pod and flowering habits. Although most of the morphological mutants were uneconomical, some mutants, nevertheless, could be used as a source of many beneficial genes in cross breeding programmes or for the improvement of some quantitative traits (Khan et al., 2011) may be useful in mapping studies (Gaur and Gaur, 2003) and in determining the evolution of the crops (Toker, 2009). Such mutants might be either a result of pleiotropic effects of mutated genes or chromosomal aberrations or gene mutations (Wani et al., 2011a). The morphological mutants differed not only in the two varieties of chickpea but also within the variety in different mutagenic treatments, suggesting that the varieties responded differently to the dose and type of mutagens employed. Based on morphological mutation frequency, Pusa-256 was proved to be the most mutable variety, while BG-1053 showed less mutability. The progenies of tall, dwarf, bushy, prostrate and bold seeded mutants bred true for the altered traits in $M_{3}$ generation. Several workers have reported that such mutant types were monogenic recessive (Jana, 1963; Sharma and Sharma, 1979; Reddy and Gupta, 1988; Satyanarayana et al., 1989; Singh et al., 1999; Talukdar, 2009). Konzak et al. (1969) in wheat and Shakoor et al. (1978) in triticale reported that semi dwarf character was controlled by polygenes. Qin et al. (2008) reported a dominant dwarf mutation, controlled by a single dominant gene in rice. EMS gave highest mutation frequency in both varieties. A high frequency and broad spectrum of morphological mutants induced by chemical mutagens has been reported in Vigna mungo (Arulbalachandran et al., 2009; Goyal and Khan, 2010b), Lens culinaris (Tyagi and Gupta, 1991; Tripathy and Dubey, 1992; Solanki and Sharma, 1999), Cicer arietinum (Khan et al., 2004a) and Glycine max (Khan and Tyagi, 2010b). Relative differences in the mutability of genes for different traits have been observed, as some of the mutant types appeared with higher frequencies in some mutagen treatments. The more frequent induction of certain mutation types by a particular mutagen may be attributed to the fact that the genes for these traits are probably more responsive to different mutagens with different modes of action. Nilan (1967) reported that different mutagens and treatment procedures might also change the relative proportion of different mutation types. Like chlorophyll mutations, combination treatments of gamma rays and EMS produced a negative synergistic effect on morphological mutations frequencies. Sharma (1970) in barley observed a synergistic effect for viable mutations at low doses and additive effect at higher doses of gamma rays + EMS.

Tall mutants, as observed in the present study, were also reported earlier by Solanki et al. (2004) in lentil and Kumar et al. (2009b) and Jana (1963) in blackgram using 
different mutagens. In comparison to the control, plant height was reduced to different magnitude in dwarf mutants, as also seen earlier in barley (Sethi, 1974), grasspea (Talukdar and Biswas, 2006) and Vigna spp. (Wani et al., 2011a). Dwarfness may result from reduced internodes length and/or internodes number (Sjodin, 1971). In the present study, reduction in internode length was mainly responsible for dwarfness. The mutant plants, which exhibited prostrate growth habit, had long internodes and weak stem. Prostrate mutants have been reported in Phaseolus vulgaris (Marghitu, 1972) and Vigna radiata (Wani et al., 2011a). Bold seeded mutant is a useful variation and can be exploited in increasing the number of seeds per pod and seed size leading to increased genetic potential of the yield. Cytology of bold seeded mutants revealed normal meiotic divisions and 8 bivalents at metaphase-I. The present bold seeded mutants may be the result of gene mutations. Singh (1996) characterized bold seeded mutants in Vigna mungo as gene mutations as there were no visible chromosomal changes associated with them. A number of early maturing mutants were isolated at various mutagen treatments in the two varieties of chickpea. In these mutants, earliness was combined with normal seed yield. However, early mutants with altered agronomic characteristics like yield and growth habit were isolated in Vigna mungo (Thakur and Sethi, 1993; Kumar et al., 2009b). Earliness is one of those characters of a crop that can be obtained reliably in mutation experiments (Gottschalk and Wolff, 1983a). Many such morphological mutants have been studied in different crop plants and attributed to the chromosomal breakage, disturbed auxin synthesis, disruption of mineral metabolism and accumulation of free amino acids (Gunkel and Sparrow, 1961; Blixt, 1972; Sharma and Sharma, 1979; Toker and Cagirgan, 2004a; Goyal and Khan, 2010b). Flower colour mutants can be exploited as genetic markers in different breeding experiments (Data and Senegupta, 2002; Atta et al., 2003).

Morphological mutants were assayed for nitrate reductase activity (NRA), total chlorophyll and carotenoid contents, which differed from the control. The role of NRA and chlorophyll contents in promoting the growth and enriching the nutritional quality of the crops through induced mutagenesis have been reported in chickpea (Barshile et al., 2009; Barshile and Apparoa, 2009), makhana (Verma et al., 2010) and Vigna spp. (Kozgar et al., 2011). In the present study, the isolated dwarf, bushy, early maturing and bold seeded mutants exhibited the higher values for total carotenoid contents with respect to the controls, depicting their role in stress physiology. A dwarf mutant of wheat, named s-dwarf, tolerant to varying level of stresses, was reported by Zhang et al. (2005). In higher plants, carotenoids protect the photosynthetic apparatus from excess of photons and oxidative stress, which are generated under stress (Siefermann-Harms. 1987; Panda and Biswal, 1989; Srichandran et al., 1989; Demmig-Adams. 1990; Youmg, 1991).

Though, it is not easy to eliminate the negative traits of this pleiotropic spectrum from the positive ones, the pleiotropic pattern of mutant gene can be altered to some extent by transferring it into a specific genotypic background (Sidorova, 1981). 